\title{
Exploring ion homeostasis and mechanism of salinity tolerance in primary tritipyrum lines (Wheat $\times$ Thinopyrum bessarabicum) in the presence of salinity
}

\author{
Maryam Kamyab ${ }^{1}$, Mohammad Kafi*1ㅜ, Hossein Shahsavand ${ }^{2}$, Morteza Goldani ${ }^{1}$, Farhad \\ Shokouhifar ${ }^{3}$
}

${ }^{1}$ Department of Agronomy and Crop Breeding, Ferdowsi University of Mashhad, Iran

${ }^{2}$ Department of Agronomy and Plant Breeding, College of Agriculture, Shiraz University, Iran

${ }^{3}$ Research Institute for Plant Sciences, Ferdowsi University, Mashhad, Iran

*Corresponding author: m.kafi@um.ac.ir

\begin{abstract}
Due to lack of water resources in irrigated agriculture, genetically improving plants to abiotic stresses such as salinity is a necessity for food and feed production. In this respect, the new third man-made amphiploid cereal, tritipyrum $\left(2 n=6 x=42, A A B B E^{b} E^{b}\right)$, is an example which is capable of tolerating a high level of $\mathrm{NaCl}$. In order to determine the salinity tolerance mechanisms of this new cereal, an experiment was conducted using hydroponic technique. Ten tritipyrum lines and two wheat cultivars were tested under three levels of salinity $(50,100$ and $200 \mathrm{mM} \mathrm{NaCl})$. The effect of salinity stress on $\mathrm{Na}^{+}$and $\mathrm{K}^{+}$concentration of leaf, shoot and root, proline and chlorophyll content were measured at $50 \%$ ear emergence and their grain yield plant ${ }^{-1}$ was evaluated at physiological maturity. Leaf $\mathrm{Na}^{+}$concentration in tritipyrum lines increased with increasing salinity while $\mathrm{K}^{+}$concentration did not show any especial pattern. The chlorophyll and proline content in tritipyrum lines were higher than that of wheat cultivars. Despite the high sodium concentration in tritipyrum lines in comparison with wheat, the grain yield of tritipyrum lines were less affected than that of wheat. There was also a negative correlation between proline content and grain yield plant ${ }^{-1}$ in tritipyrum lines. It can be concluded that mechanisms such as higher $\mathrm{Na}^{+}$uptake along with appropriate ion compartmentation could be used by tritipyrum lines to combat with salt stress like some halophytes and it can make tritipyrum lines suitable for planting in saline soils and improving the salinity tolerance of wheat.
\end{abstract}

Keywords: Halophytic wild ancestors, Proline, Salt tolerance mechanism, Tritipyrum.

Abbreviations: $\mathrm{LNa}_{-}$leaf sodium concentration; $\mathrm{LK}_{-}$leaf potassium concentration; $\mathrm{LK}: \mathrm{Na}$ or $\mathrm{L} \mathrm{K}^{+} / \mathrm{Na}^{+} \_$leaf potassium to sodium ratio; $\mathrm{SN}_{-}$stem sodium concentration; $\mathrm{SK}_{-}$stem potassium concentration; $\mathrm{SK}: \mathrm{Na}_{-}$stem potassium to sodium ratio; $\mathrm{RN}_{-}$root sodium concentration; $\mathrm{RK}_{-}$root potassium concentration; $\mathrm{RK}: \mathrm{Na}$ _ root potassium to sodium ratio; Chl a_chlorophyll $a$ concentration; $\mathrm{Chl} \mathrm{b}_{-}$chlorophyll $\mathrm{b}$ concentration; $\mathrm{Chl} a / b_{-}$chlorophyll $a$ to chlorophyll $b$ ratio; $\mathrm{T}$ Chl_ total chlorophyll; GY_ grain yield plant ${ }^{-1}$; Pr: proline content; $\mathrm{DW}_{-}$dry weight; FW_ fresh weight; PCA_ principle component analysis; Yp_ yield of genotype in stress condition; $\mathrm{Yp}_{-}$yield of genotypes under control condition; $\mathrm{Na}^{+}{ }_{-}$sodium concentration and $\mathrm{K}^{+}$- potassium concentration.

\section{Introduction}

Despite the favorable effects of wheat domestication such as increasing the quality and quantity of yield, it has created some genetic bottlenecks such as genetic erosion. This by itself leads to an increased susceptibility to some environmental stresses like salinity (that currently affects around $\% 7$ of the earth's surface). Therefore, the need for another neo-domestication of wheat was felt (Faris, 2014; Peng et al., 2011). To achieve this goal and improve salinity tolerance of wheat, use of genetic resources such as its halophytic wild ancestors like tall wheat grass spp (e.g. Thinopyrum spp.) is suggested (Colmer et al., 2006a; Colmer et al., 2006b; Peng et al., 2011). Among tall wheat grasses, Thinopyrum bessarabicum (a salt-tolerant perennial wheat grass that grows on sea shores of Crimea) can survive up to $350 \mathrm{mM} \mathrm{NaCl}$ for long periods (Gorham et al., 1985). Maintaining leaf $\mathrm{K}^{+}$concentration, synthesizing glycinebetaine and regulating leaf $\mathrm{Na}^{+}$and $\mathrm{Cl}^{-}$concentration for osmotic adjustment are strategies that have been adopted by Th. bessarabicum to resist salinity (Colmer et al., 2006b).
Hybridization between wheat and Th. Bessarabicumhas shown encouraging results and has resulted in the creation of a third man-made amphiploid (tritipyrum) (King et al., 1997). It is a new product that is promising the production of a new synthetic amphiploid like triticale and it has $E^{\mathrm{b}}$ genome instead of D genome in hexaploid wheat. This amphiploid has high tolerance to salinity, wheat like growth, seed set and novel multiple seed trait in some lines and its meiosis is generally regular. Of course it has undesirable traits such as late maturity, continuous production of tillers, brittle rachis and partial meiotic instability. It is capable of becoming a new crop and it has been suggested to be used as a new cereal crop in saline soils (King et al., 1997). However, a question that is raised about tritipyrum is that: among salinity resistance mechanisms which ones are used by tritipyrum lines?

During salinity stress, $\mathrm{Na}^{+}$not only takes over $\mathrm{Na}^{+} / \mathrm{K}^{+}$cotransporters, but it can also block $\mathrm{K}^{+}$specific transporters and these events cause increasing sodium entree up to toxic 
levels, and decreasing potassium uptake until there is insufficient concentration for enzymatic reaction and osmotic adjustment (Apse and Blumwald, 2007). Also salinity stress changes photosynthetic pathway, decreases chlorophyll content and increases chlorophyll $a /$ chlorophyll $b$ ratio (Parida and Das, 2005; Khan, 2003; Khatkar and Kuhad, 2000). More than one salt resistance mechanism is involved in plants and a combination of factors and strategies contributes to wheat salinity resistance (Flowers and Colmer, 2008; Mudgal et al., 2010; Munns and Tester, 2008). Tolerance to osmotic stress, $\mathrm{Na}^{+}$exclusion from leaf blade and tissue tolerance were introduced as mechanisms of salinity tolerance (Munns, 2008). Compartmentation of $\mathrm{Na}^{+}$ and increasing accumulation of compatible solutes like proline are strategies that are used by tissue tolerance mechanisms (Munns, 2008). Some studies have shown a good relation between $\mathrm{Na}^{+}$exclusion and salt tolerance (Poustini and Siosemardeh, 2004; Munns and Jame, 2003). However, in other studies no significant relation between $\mathrm{Na}^{+}$ exclusion and salt tolerance has been found (Genc, 2007). Accumulation of $\mathrm{Na}^{+}$in the vacuole disturbs the balance of osmotic pressure of ions; hence collection of organic solutes like proline in cytosol controls this disorder (Munns, 2008). Thus, this parameter has been supported as a criterion of salt stress tolerance (Ashraf and Harris, 2004). However, some researchers have found that there is no correlation between leaf proline and salt tolerance (Poustini et al, 2007; Lutts et al., 1996).

The aims of the present study were as follows. Firstly comparing salinity resistance, ion homeostasis, proline content as well as chlorophyll content of primary tritipyrum lines with bread wheat cultivars, Secondly, what is the effect of these parameters on the resistance of primary tritipyrum lines to salinity in controlled environment?, and Thirdly, to determine whether $\mathrm{Na}^{+}$exclusion or tissue tolerance cause ion homeostasis in primary tritipyrum lines.

\section{Results}

Out of 10 primary tritipyrum lines and two wheat cultivars, the wheat cultivar Gascoigne (salt sensitive) lacked enough tolerance and died due to salinity stress. Moreover, one of the primary tritipyrum lines $(\mathrm{St} / \mathrm{b})$ displayed late maturity and stayed green up to the end of experiment. Therefore, these two genotypes were eliminated from the analysis.

\section{$\mathrm{Na}^{+}$and $\mathrm{K}^{+}$ion concentration in different tissues}

The flag leaf $\mathrm{Na}^{+}$concentration pattern over three levels of salinity $(50,100$ and $200 \mathrm{mM} \mathrm{NaCl})$ was similar and an increase in flag leaf $\mathrm{Na}^{+}$accumulation with increasing salinity levels was observed in all tested genotypes (Table2). In primary tritipyrum lines except on $\mathrm{La}(4 \mathrm{~B}, 4 \mathrm{D}) / \mathrm{b}$, flag leaf $\mathrm{Na}^{+}$concentration increased highly from 50 to $100 \mathrm{mM}$, but no tangible increase was observed from $100 \mathrm{mM}$ to the highest salinity level (200 mM) (Table 2). On the other hand, $\mathrm{Na}^{+}$concentration showed an intense increase in wheat cultivar Bam from $50 \mathrm{mM}$ to $200 \mathrm{mM}$ (Table 2). The flag leaf $\mathrm{Na}^{+}$concentration in wheat cultivar Bam was lower than that of tritipyrum lines at $50 \mathrm{mM}$, but there was a 25 -fold difference in $\mathrm{Na}^{+}$concentration of wheat at 50 compared to $200 \mathrm{mM}$, while the value of primary tritipyrum lines was approximately equal to 2.5 -fold (Table 2 ). At the highest salinity level $(200 \mathrm{mM})$, the flag leaf of $(\mathrm{Ka} / \mathrm{b}) *(\mathrm{Cr} / \mathrm{b}), \mathrm{F}_{6}$ line showed the lowest, whereas $\mathrm{La}(4 \mathrm{~B}, 4 \mathrm{D}) / \mathrm{b}$ showed the highest $\mathrm{Na}^{+}$accumulation (Table 2). In the case of stem, $\mathrm{NaCl}$ treatment caused an increase in $\mathrm{Na}^{+}$concentration in all genotypes (Table 2). Genotypes exhibited little variation in stem $\mathrm{Na}^{+}$concentration from 50 to $200 \mathrm{mM} \mathrm{NaCl}$, with the exception of $\mathrm{Ka} / \mathrm{b}$ line and wheat cultivar Bam. At $50 \mathrm{mM}$ $\mathrm{NaCl}, \mathrm{Ka} / \mathrm{b}$ line had low $\mathrm{Na}^{+}$concentration $(0.61 \mathrm{mg} / \mathrm{g} \mathrm{DW})$ and $(\mathrm{Ma} / \mathrm{b}) *(\mathrm{Cr} / \mathrm{b}), \mathrm{F}_{4}$ had high $\mathrm{Na}^{+}$concentration $(2.7 \mathrm{mg} / \mathrm{g}$ DW) (Table 2). At the highest level of salinity, maximum $\mathrm{Na}^{+}$concentration was related to $\mathrm{La} / \mathrm{b}$ and minimum value was associated with $(\mathrm{Ka} / \mathrm{b}) *(\mathrm{Cr} / \mathrm{b}), \mathrm{F}_{2}$ (Table 2$)$. In primary tritipyrum lines, the rate of stem $\mathrm{Na}^{+}$accumulation was constant while, it was higher in wheat cultivar Bam (Table 2). $\mathrm{Na}^{+}$concentration in root tissue increased at all salinity levels, but the variation among genotypes was outstanding (Table 2). At the highest salinity level $(200 \mathrm{mM})$, maximum root $\mathrm{Na}^{+}$concentration was related to $\mathrm{La}(4 \mathrm{~B}, 4 \mathrm{D}) / \mathrm{b}$ and $\mathrm{Az} / \mathrm{b}$ and minimum value was associated with $\mathrm{Ka} / \mathrm{b}$ (Table 2 ).

There was no specific trend for $\mathrm{K}^{+}$concentration of flag leaf in all genotypes at different salinity levels (Table 2). However, it was noteworthy that in wheat cultivar Bam, fluctuation of $\mathrm{K}^{+}$concentration of flag leaf at different salinity levels was more than tritipyrum lines (Table 2 ). The flag leaf $\mathrm{K}^{+}$concentration of $(\mathrm{Ka} / \mathrm{b}) *(\mathrm{Cr} / \mathrm{b}), \mathrm{F}_{6}$ line with low flag leaf $\mathrm{Na}^{+}$accumulation was lower than that of other varieties at the highest salinity level (Table 2 ). Similar to $\mathrm{K}^{+}$ concentration of flag leaf, there was no clear trend in stem $\mathrm{K}^{+}$ concentration during salinity stress (Table 2 ). At the highest salinity level, $\mathrm{Ka} / \mathrm{b}$ had the highest leaf $\mathrm{K}^{+}$concentration (Table 2). Also, there was no distinct pattern for root $\mathrm{K}^{+}$ concentration and wheat cultivar Bam showed the highest $\mathrm{K}^{+}$ concentration at all salinity levels compared to primary tritipyrum lines except at $50 \mathrm{mM} \mathrm{NaCl}$ (Table 2). Generally, both primary tritipyrum lines and wheat cultivar Bam showed a decrease in $\mathrm{Na}^{+}$concentration from root to flag leaf at all salinity levels while this trend was reversed in case of potassium (Fig. 1).

The genotypes differed in flag leaf $\mathrm{Ka}^{+} / \mathrm{Na}^{+}$ratio at all salinity levels (Table 2). Some primary tritipyrum lines showed higher and some showed lower $\mathrm{Ka}^{+} / \mathrm{Na}^{+}$ratios than wheat cultivar Bam (Table 2). In stem at $50 \mathrm{mM}$ and $100 \mathrm{mM}$ $\mathrm{NaCl}$, the varieties were different in terms of $\mathrm{Ka}^{+} / \mathrm{Na}^{+}$ratio. But at $200 \mathrm{mM}$, there were fewer variations among the varieties. All varieties exhibited a decrease in $\mathrm{Ka}^{+} / \mathrm{Na}^{+}$ratio with increasing salinity (Table 2). For all varieties under salt stress, root $\mathrm{Ka}^{+} / \mathrm{Na}^{+}$ratios had decreased (Table 2). At two salinity levels $(50$ and $100 \mathrm{mM} \mathrm{NaCl}$ ) wheat cultivar Bam had the highest $\mathrm{Ka}^{+} / \mathrm{Na}^{+}$ratio (Table 2).

\section{Salinity and photosynthetic pigment concentration}

Increasing the external salinity level up to $100 \mathrm{mM}$ led to an increase in chlorophyll $a$ content, but it decreased at $200 \mathrm{mM}$ (Table 2). In all salinity levels, wheat cultivar Bam had lower Chl-a than primary tritipyrum lines and $(\mathrm{Ma} / \mathrm{b}) *(\mathrm{Cr} / \mathrm{b}), \mathrm{F}_{4}$ had higher Chl-a content at $100 \mathrm{mM} \mathrm{NaCl}$ (Table 2).

There was no certain pattern for chlorophyll $b$ content. The highest $\mathrm{Chl} b$ content was related to $(\mathrm{Ka} / \mathrm{b}) *(\mathrm{Cr} / \mathrm{b}), \mathrm{F}_{2}$ at 100 $\mathrm{mM}$ and the lowest one was related to $(\mathrm{Ka} / \mathrm{b}) *(\mathrm{Cr} / \mathrm{b}), \mathrm{F}_{6}$ at $200 \mathrm{mM}$ (Table 2). Generally primary tritipyrum lines exhibited higher chlorophyll $a$ and chlorophyll $b$ than wheat cultivar Bam (Table 2). 
Table 1. Abbreviation, ploidy level and genomic constitution of primary tritipyrum lines and wheat cultivars in the present study.

\begin{tabular}{|c|c|c|c|}
\hline Genotypes & Abbreviation & Ploidy & Genome \\
\hline \multicolumn{4}{|l|}{ Wheat cultivars } \\
\hline T.aestivum cv. Bam & Bam & $6 \mathrm{X}$ & AABBDD \\
\hline T.aestivum cv. Gascoigen & Gascoigen & $6 \mathrm{X}$ & AABBDD \\
\hline \multicolumn{4}{|l|}{ Tritipyrum lines } \\
\hline$\left(\right.$ Karim/Th. Bessarabicum)*( Creso/ Th. Bessarabicum) $\mathrm{F}_{6}$ & $(\mathrm{Ka} / \mathrm{b}) *(\mathrm{Cr} / \mathrm{b}), \mathrm{F}_{6}$ & $6 \mathrm{X}$ & $A A B B E^{b} E^{b}$ \\
\hline 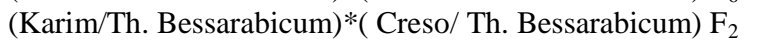 & $(\mathrm{Ka} / \mathrm{b}) *(\mathrm{Cr} / \mathrm{b}), \mathrm{F}_{2}$ & $6 \mathrm{X}$ & $A A B B E^{b} E^{b}$ \\
\hline (Macoun/ Th. Bessarabicum)*( Creso/ Th. Bessarabicum) $\mathrm{F}_{4}$ & $(\mathrm{Ma} / \mathrm{b}) *(\mathrm{Cr} / \mathrm{b}), \mathrm{F}_{4}$ & $6 \mathrm{X}$ & $A A B B E^{b} E^{b}$ \\
\hline (Stewart/ Th. Bessarabicum)*( Creso/ Th. Bessarabicum) $\mathrm{F}_{4}$ & $(\mathrm{St} / \mathrm{b})^{*}(\mathrm{Cr} / \mathrm{b}), \mathrm{F}_{4}$ & $6 \mathrm{X}$ & $A A B B E^{b} E^{b}$ \\
\hline Langdon/ Th. Bessarabicum (4B,4D)/ Th. Bessarabicum & $\mathrm{La}(4 \mathrm{~B}, 4 \mathrm{D}) / \mathrm{b}$ & $6 \mathrm{X}$ & $A A B B E^{b} E^{b}$ \\
\hline Karim/Th. Bessarabicum & $\mathrm{Ka} / \mathrm{b}$ & $6 \mathrm{X}$ & $A A B B E^{b} E^{b}$ \\
\hline Creso/ Th. Bessarabicum & $\mathrm{Cr} / \mathrm{b}$ & $6 \mathrm{X}$ & $A A B B E^{b} E^{b}$ \\
\hline Stewart/ Th. Bessarabicum & $\mathrm{St} / \mathrm{b}$ & $6 \mathrm{X}$ & $A A B B E^{b} E^{b}$ \\
\hline Langdon/ Th. Bessarabicum & $\mathrm{La} / \mathrm{b}$ & $6 \mathrm{X}$ & $A A B B E^{b} E^{b}$ \\
\hline Aziziah/ Th. Bessarabicum & $\mathrm{Az} / \mathrm{b}$ & $6 X$ & $A A B B E^{b} E^{b}$ \\
\hline
\end{tabular}

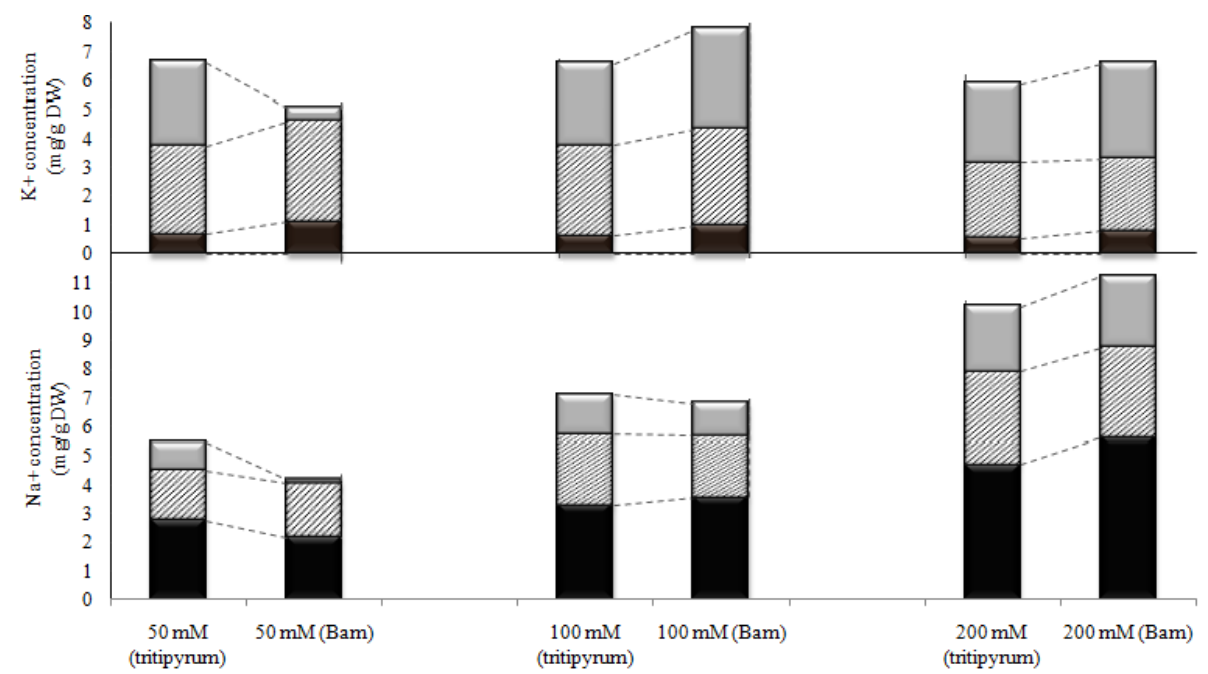

salinity level $(\mathrm{mmol} /)$

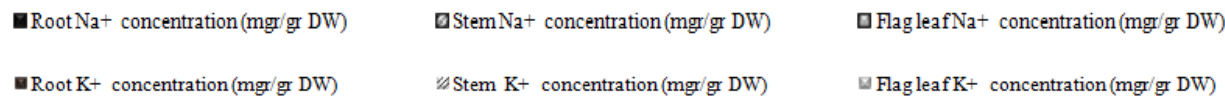

Fig 1. $\mathrm{Na}^{+}$and $\mathrm{K}^{+}$concentration in different tissue of tritipyrum lines and wheat cultivar Bam in different $\mathrm{NaCl}$ levels.

The trend for total chlorophyll content and $\mathrm{Chl} a / b$ ratio of the genotypes under salt stress was similar to Chl-a (Table 2). In relation to $\mathrm{Chl} a / b$ ratio, $(\mathrm{Ka} / \mathrm{b}) *(\mathrm{Cr} / \mathrm{b}), \mathrm{F}_{6}$ had the highest amount of $\mathrm{Chl} a / b$ ratio at all salinity levels.

\section{Proline content and salinity}

Salt treatment influenced proline content of primary tritipyrum lines and wheat flag leaves (Fig. 2). Increasing salinity level caused increasing proline content of wheat cultivar Bam leavesbut in tritipyrum lines, increasing salinity from $50 \mathrm{mM}$ to $100 \mathrm{mM}$ caused a decrease in proline content but it was not remarkable. However, at $200 \mathrm{mM}$ salinity a marked increase in proline content of tritipyrum leaves were induced (Fig. 2). In $50 \mathrm{mM} \mathrm{NaCl}$ treatment the lowest proline content belonged to wheat cultivar Bam (Fig. 2). On the average, the proline conent of tritipyrum lines was approximately 1.5 fold more than its content in wheat cultivar Bam.

\section{Grain yield under salt stress treatment}

Primary tritipyrum lines could demonstrate a better performance against salt stress than wheat cultivar Bam and their grain yield was more than Bam particularly at 100 and $200 \mathrm{mM} \mathrm{NaCl}$ levels (Fig.3). At the first level of salt treatment $(50 \mathrm{mM})$, grain yield of primary tritipyrum lines was similar to wheat cultivar Bam. Except on $\mathrm{Ka} / \mathrm{b}$ and $\mathrm{Az} / \mathrm{b}$, other primary tritipyrum lines produced similar grain yield or even lower grain yield in comparison with wheat cultivar Bam. However, at this salt level, $\mathrm{La}(4 \mathrm{~B}, 4 \mathrm{D}) / \mathrm{b}$ had the lowest $(0.65 \mathrm{~g} / \mathrm{plant})$ and $\mathrm{Ka} / \mathrm{b}$ had the highest $(1.18 \mathrm{~g} / \mathrm{plant})$ grain yield (Fig.3). At the second salt treatment $(100 \mathrm{mM})$, grain yield of all primary tritipyrum lines except $\mathrm{La}(4 \mathrm{~B}, 4 \mathrm{D}) / \mathrm{b}$ was satisfactory, but a significant decrease was observed in yield of wheat cultivar Bam. It was noteworthy that primary tritipyrum lines grain yield in $100 \mathrm{mM}$ was higher than Bam grain yield (Fig. 3). At $100 \mathrm{mM}$, only two lines $\mathrm{La} \mathrm{(4B,} \mathrm{4D)/b}$ and $\mathrm{Cr} / \mathrm{b}$ produced lower grain yield than wheat cultivar $\mathrm{Bam}$. The highest grain yield belonged to $(\mathrm{St} / \mathrm{b}) *(\mathrm{Cr} / \mathrm{b}), \mathrm{F}_{4}$ 
Table 2. Ion concentration and $\mathrm{K}^{+} / \mathrm{Na}^{+}$ratio in flag leaf, stem and root of primary tritipyrum lines and bread wheat (cv;Bam) under different salinity levels.

\begin{tabular}{|c|c|c|c|c|c|c|c|c|c|c|c|c|c|c|}
\hline \multirow{4}{*}{$\begin{array}{c}\text { genotypes } \\
(\mathrm{Ka} / \mathrm{b})^{*}(\mathrm{Cr} / \mathrm{b}), \mathrm{F}_{2}\end{array}$} & \multirow{3}{*}{$\begin{array}{c}\begin{array}{c}\text { Salinit } \\
y \\
\text { level } \\
(\mathrm{mM})\end{array} \\
\end{array}$} & \multicolumn{3}{|c|}{$\begin{array}{l}\mathrm{Na}^{+} \text {concentration } \\
(\mathrm{mg} / \mathrm{g} \mathrm{DW})\end{array}$} & \multicolumn{3}{|c|}{$\begin{array}{c}\mathrm{K}^{+} \text {concentration } \\
(\mathrm{mg} / \mathrm{g} \mathrm{DW})\end{array}$} & \multicolumn{3}{|c|}{$\mathrm{K}^{+} / \mathrm{Na}^{+}$ratio } & \multirow{2}{*}{$\begin{array}{c}\text { Chlorop } \\
\text { hyll a } \\
\text { (mg/g } \\
\text { FW) }\end{array}$} & \multirow{2}{*}{$\begin{array}{c}\text { Chloro } \\
\text { phyll b } \\
\text { (mg/g } \\
\text { FW) }\end{array}$} & \multirow{2}{*}{$\begin{array}{l}\text { Chloro } \\
\text { phyll a/ } \\
\text { chlorop } \\
\text { hyll b } \\
\text { Chl } \\
\text { a/Chl b }\end{array}$} & \multirow{2}{*}{$\begin{array}{c}\begin{array}{c}\text { Total } \\
\text { chlorop } \\
\text { hyll }\end{array} \\
\text { Total } \\
\text { Chl } \\
\end{array}$} \\
\hline & & Leaf & Stem & Root & Leaf & Stem & Root & Leaf & Stem & Root & & & & \\
\hline & & $0.55^{\mathrm{gh} *}$ & $0.90^{\mathrm{jk}}$ & $2.95^{\mathrm{dk}}$ & $2.70^{\mathrm{bd}}$ & $3.09^{\mathrm{ch}}$ & $0.71^{\text {ch }}$ & $5.08^{\mathrm{ac}}$ & $3.46^{b}$ & $0.24^{\mathrm{bg}}$ & $1.49^{\mathrm{a}}$ & $0.58 \mathrm{ac}$ & $2.62 \mathrm{bc}$ & $2.06^{\mathrm{ab}}$ \\
\hline & 100 & $1.53^{\mathrm{dg}}$ & $1.75^{\mathrm{hj}}$ & $3.08^{\mathrm{dk}}$ & $2.64^{\mathrm{bd}}$ & $3.96^{\mathrm{ab}}$ & $0.72^{\mathrm{cg}}$ & $1.76^{\mathrm{hm}}$ & $2.31^{\mathrm{c}}$ & $0.24^{\text {bh }}$ & $1.52^{\mathrm{a}}$ & $0.77 \mathrm{a}$ & $2.04 \mathrm{c}$ & $2.29^{\mathrm{a}}$ \\
\hline & 200 & $2.11^{\text {be }}$ & $2.34^{\mathrm{eh}}$ & $3.84^{\mathrm{cd}}$ & $2.61^{\mathrm{bd}}$ & $2.31^{\mathrm{hi}}$ & $0.34^{\mathrm{hj}}$ & $1.27^{\mathrm{jm}}$ & $1.01^{\mathrm{fg}}$ & $0.09^{\mathrm{ik}}$ & $1.45^{\mathrm{a}}$ & $0.56 \mathrm{ac}$ & $2.67 \mathrm{bc}$ & $2.01^{\mathrm{ab}}$ \\
\hline \multirow[t]{3}{*}{$(\mathrm{Ka} / \mathrm{b})^{*}(\mathrm{Cr} / \mathrm{b}), \mathrm{F}_{6}$} & 50 & $0.50^{\text {gh }}$ & $1.00^{\mathrm{jk}}$ & $2.69^{\mathrm{ek}}$ & $3.01^{\mathrm{ad}}$ & $3.23^{\text {be }}$ & $0.73^{\mathrm{bg}}$ & $6.33^{\mathrm{a}}$ & $3.25^{b}$ & $0.27^{\text {bf }}$ & $1.33^{\mathrm{a}}$ & $0.40^{\mathrm{bc}}$ & $3.30^{\mathrm{ac}}$ & $1.73^{\mathrm{ab}}$ \\
\hline & 100 & $0.51^{\text {gh }}$ & $1.40^{\mathrm{ik}}$ & $2.92^{\mathrm{dk}}$ & $2.91^{\mathrm{bd}}$ & $3.12^{\mathrm{cg}}$ & $0.63^{\mathrm{ci}}$ & $6.19^{\mathrm{ab}}$ & $2.27^{\mathrm{c}}$ & $0.22^{\text {bi }}$ & $1.40^{\mathrm{a}}$ & $0.38^{\mathrm{bc}}$ & $3.77^{\mathrm{ab}}$ & $1.78^{\mathrm{ab}}$ \\
\hline & 200 & $0.56^{\mathrm{gh}}$ & $2.56^{\mathrm{dh}}$ & $3.84^{\mathrm{cd}}$ & $2.06^{\mathrm{cd}}$ & $2.71^{\mathrm{ci}}$ & $0.64^{\mathrm{ci}}$ & $3.68^{\mathrm{cf}}$ & $1.06^{\mathrm{fg}}$ & $0.17^{\mathrm{ek}}$ & $1.37^{\mathrm{a}}$ & $0.33^{c}$ & $4.37^{\mathrm{a}}$ & $1.70^{\mathrm{ab}}$ \\
\hline \multirow[t]{3}{*}{$(\mathrm{Ma} / \mathrm{b})^{*}(\mathrm{Cr} / \mathrm{b}), \mathrm{F}_{4}$} & 50 & $1.23^{\mathrm{eg}}$ & $2.70^{\mathrm{dg}}$ & $2.82^{\mathrm{ek}}$ & $3.91^{\mathrm{ab}}$ & $2.56^{\mathrm{di}}$ & $0.47^{\mathrm{ej}}$ & $3.18^{\mathrm{dh}}$ & $0.95^{\mathrm{fg}}$ & $0.17^{\mathrm{ek}}$ & $1.48^{\mathrm{a}}$ & $0.55^{\mathrm{ac}}$ & $2.86^{\mathrm{ac}}$ & $2.03^{\mathrm{ab}}$ \\
\hline & 100 & $1.46^{\mathrm{eg}}$ & $2.84^{\mathrm{cg}}$ & $3.48^{\mathrm{cg}}$ & $3.14^{\mathrm{ad}}$ & $3.21^{\mathrm{bf}}$ & $0.30^{\mathrm{ij}}$ & $2.32^{\mathrm{em}}$ & $1.13^{\mathrm{fg}}$ & $0.09^{\mathrm{ik}}$ & $1.54^{\mathrm{a}}$ & $0.56^{\mathrm{ac}}$ & $2.78^{\mathrm{ac}}$ & $2.11^{\mathrm{ab}}$ \\
\hline & 200 & $2.66^{\mathrm{bc}}$ & $3.66^{\mathrm{ac}}$ & $5.30^{\mathrm{a}}$ & $2.93^{b d}$ & $2.50^{\mathrm{ei}}$ & $0.47^{\mathrm{ej}}$ & $1.12^{\mathrm{lm}}$ & $0.69^{\mathrm{g}}$ & $0.09^{\mathrm{ik}}$ & $1.26^{\mathrm{a}}$ & $0.45^{\mathrm{ac}}$ & $2.83^{\mathrm{ac}}$ & $1.72^{a b}$ \\
\hline \multirow[t]{3}{*}{$(\mathrm{St} / \mathrm{b})^{*}(\mathrm{Cr} / \mathrm{b}), \mathrm{F}_{4}$} & 50 & $0.96^{\text {fh }}$ & $2.37^{\mathrm{eh}}$ & $3.60^{\mathrm{ce}}$ & $2.86^{\text {bd }}$ & $3.42^{\mathrm{ac}}$ & $0.52^{\mathrm{dj}}$ & $3.11^{\mathrm{di}}$ & $1.46^{\mathrm{cg}}$ & $0.15^{\mathrm{fk}}$ & $1.48^{\mathrm{a}}$ & $0.48^{\mathrm{ac}}$ & $3.19^{\mathrm{ac}}$ & $1.95^{\mathrm{ab}}$ \\
\hline & 100 & $1.57^{\mathrm{cg}}$ & $2.90^{\mathrm{cg}}$ & $3.59^{\mathrm{cf}}$ & $2.71^{\mathrm{bd}}$ & $4.21^{\mathrm{a}}$ & $0.78^{\text {af }}$ & $1.73^{\mathrm{hm}}$ & $1.47^{\mathrm{cg}}$ & $0.22^{\mathrm{bi}}$ & $1.53^{\mathrm{a}}$ & $0.66^{\mathrm{ac}}$ & $2.34^{\mathrm{bc}}$ & $2.19^{\mathrm{a}}$ \\
\hline & 200 & $1.71^{\mathrm{bf}}$ & $3.35^{\mathrm{ad}}$ & $5.08^{\mathrm{ab}}$ & $2.61^{\mathrm{bd}}$ & $2.66^{\mathrm{ci}}$ & $0.40^{\mathrm{gj}}$ & $1.52^{\mathrm{im}}$ & $0.79^{\mathrm{fg}}$ & $0.08^{\mathrm{jk}}$ & $1.48^{\mathrm{a}}$ & $0.59^{\mathrm{ac}}$ & $2.58^{\mathrm{bc}}$ & $2.07^{\mathrm{ab}}$ \\
\hline \multirow[t]{3}{*}{$\mathrm{La}(4 \mathrm{~B}, 4 \mathrm{D}) / \mathrm{b}$} & 50 & $1.75^{\text {bf }}$ & $1.76^{\mathrm{hj}}$ & $2.23^{\mathrm{jk}}$ & $3.13^{\mathrm{ad}}$ & $2.40^{\mathrm{fi}}$ & $0.67^{\mathrm{ci}}$ & $1.80^{\mathrm{hm}}$ & $1.37^{\mathrm{dg}}$ & $0.30^{\text {bd }}$ & $1.36^{\mathrm{a}}$ & $0.51^{\mathrm{ac}}$ & $2.72^{\mathrm{ac}}$ & $1.87^{\mathrm{ab}}$ \\
\hline & 100 & $1.81^{\mathrm{bf}}$ & $2.24^{\mathrm{fi}}$ & $3.31^{\mathrm{ci}}$ & $3.22^{\mathrm{ad}}$ & $2.10^{\mathrm{i}}$ & $0.71^{\text {ch }}$ & $1.81^{\mathrm{hm}}$ & $0.94^{\mathrm{fg}}$ & $0.21^{\mathrm{bi}}$ & $1.42^{\mathrm{a}}$ & $0.42^{\mathrm{bc}}$ & $3.41^{\mathrm{ac}}$ & $1.84^{\mathrm{ab}}$ \\
\hline & 200 & $5.40^{\mathrm{a}}$ & $3.20^{\mathrm{ae}}$ & $5.79^{a}$ & $4.49^{\mathrm{a}}$ & $2.38^{\mathrm{gi}}$ & $0.64^{\mathrm{ci}}$ & $0.84^{\mathrm{m}}$ & $0.76^{\mathrm{fg}}$ & $0.11^{\mathrm{gk}}$ & $1.10^{\mathrm{a}}$ & $0.38^{\mathrm{bc}}$ & $2.92^{\mathrm{ac}}$ & $1.48^{b}$ \\
\hline \multirow[t]{3}{*}{$\mathrm{Az} / \mathrm{b}$} & 50 & $0.61^{\text {gh }}$ & $1.43^{\mathrm{ik}}$ & $2.37^{\mathrm{ik}}$ & $2.81^{\text {bd }}$ & $3.01^{\mathrm{ch}}$ & $1.15^{\mathrm{a}}$ & $4.59^{\mathrm{bd}}$ & $2.13^{\mathrm{cd}}$ & $0.48^{\mathrm{a}}$ & $1.42^{\mathrm{a}}$ & $0.60^{\mathrm{ac}}$ & $2.39^{\mathrm{bc}}$ & $2.02^{\mathrm{ab}}$ \\
\hline & 100 & $0.98^{\text {fh }}$ & $2.07^{\mathrm{gi}}$ & $3.39^{\mathrm{ch}}$ & $2.70^{\mathrm{bd}}$ & $3.23^{\text {be }}$ & $0.87^{\mathrm{ad}}$ & $2.73^{\mathrm{ek}}$ & $1.59^{\mathrm{cf}}$ & $0.25^{\mathrm{bf}}$ & $1.49^{\mathrm{a}}$ & $0.56^{\mathrm{ac}}$ & $2.75^{\mathrm{ac}}$ & $2.04^{\mathrm{ab}}$ \\
\hline & 200 & $1.22^{\mathrm{eg}}$ & $3.00^{\mathrm{bf}}$ & $5.59^{\mathrm{a}}$ & $2.50^{\mathrm{bd}}$ & $2.68^{\mathrm{ci}}$ & $0.84^{\text {ae }}$ & $2.07^{\mathrm{gm}}$ & $0.90^{\mathrm{fg}}$ & $0.15^{\mathrm{ek}}$ & $1.28^{\mathrm{a}}$ & $0.50^{\mathrm{ac}}$ & $2.66^{\mathrm{bc}}$ & $1.78^{\mathrm{ab}}$ \\
\hline \multirow[t]{3}{*}{$\mathrm{Cr} / \mathrm{b}$} & 50 & $1.39^{\mathrm{eg}}$ & $2.51^{\mathrm{dh}}$ & $2.46^{\mathrm{hk}}$ & $3.05^{\mathrm{ad}}$ & $3.12^{\mathrm{cg}}$ & $0.41^{\mathrm{fj}}$ & $2.25^{\mathrm{fm}}$ & $1.26^{\mathrm{eg}}$ & $0.17^{\mathrm{dk}}$ & $1.20^{\mathrm{a}}$ & $0.59^{\mathrm{ac}}$ & $2.02^{c}$ & $1.79^{\mathrm{ab}}$ \\
\hline & 100 & $1.57^{\mathrm{cg}}$ & $3.00^{\text {bf }}$ & $3.55^{\mathrm{cg}}$ & $2.77^{\text {bd }}$ & $2.82^{\mathrm{ci}}$ & $0.38^{\mathrm{gj}}$ & $1.82^{\mathrm{hm}}$ & $0.95^{\mathrm{fg}}$ & $0.11^{\mathrm{hk}}$ & $1.36^{\mathrm{a}}$ & $0.58^{\mathrm{ac}}$ & $2.37^{\mathrm{bc}}$ & $1.94^{\mathrm{ab}}$ \\
\hline & 200 & $2.69^{b}$ & $3.63^{\mathrm{ac}}$ & $5.31^{\mathrm{a}}$ & $2.55^{\text {bd }}$ & $2.86^{\mathrm{ci}}$ & $0.25^{\mathrm{j}}$ & $0.98^{\mathrm{Im}}$ & $0.79^{\mathrm{fg}}$ & $0.05^{\mathrm{k}}$ & $1.27^{\mathrm{a}}$ & $0.57^{\mathrm{ac}}$ & $2.26^{\mathrm{bc}}$ & $1.84^{\mathrm{ab}}$ \\
\hline \multirow[t]{3}{*}{$\mathrm{Ka} / \mathrm{b}$} & 50 & $0.54^{\mathrm{gh}}$ & $0.61^{\mathrm{k}}$ & $2.64^{\mathrm{fk}}$ & $1.93^{\mathrm{de}}$ & $2.91^{\mathrm{ci}}$ & $0.83^{\text {ae }}$ & $3.60^{\mathrm{cg}}$ & $4.88^{\mathrm{a}}$ & $0.31^{\mathrm{bc}}$ & $1.40^{\mathrm{a}}$ & $0.69^{a b}$ & $2.05^{\mathrm{c}}$ & $2.10^{\mathrm{ab}}$ \\
\hline & 100 & $2.08^{\text {be }}$ & $2.80^{\mathrm{cg}}$ & $2.62^{\mathrm{gk}}$ & $2.70^{\mathrm{bd}}$ & $2.83^{\mathrm{ci}}$ & $0.54^{\mathrm{dj}}$ & $1.32^{\mathrm{jm}}$ & $1.01^{\mathrm{fg}}$ & $0.21^{\mathrm{cj}}$ & $1.46^{\mathrm{a}}$ & $0.63^{\mathrm{ac}}$ & $2.34^{\mathrm{bc}}$ & $2.08^{a b}$ \\
\hline & 200 & $2.60^{\mathrm{bd}}$ & $3.12^{b f}$ & $3.02^{\mathrm{dk}}$ & $2.94^{\mathrm{bd}}$ & $3.16^{\mathrm{bg}}$ & $0.84^{\text {ae }}$ & $1.14^{\mathrm{km}}$ & $1.01^{\mathrm{fg}}$ & $0.28^{\text {be }}$ & $1.36^{\mathrm{a}}$ & $0.61^{\mathrm{ac}}$ & $2.25^{\mathrm{bc}}$ & $1.97^{\mathrm{ab}}$ \\
\hline \multirow[t]{3}{*}{$\mathrm{La} / \mathrm{b}$} & 50 & $1.24^{\mathrm{eg}}$ & $2.42^{\text {eh }}$ & $3.14^{\mathrm{dj}}$ & $3.47^{\mathrm{ad}}$ & $3.35^{\mathrm{bd}}$ & $0.56^{\mathrm{cj}}$ & $2.83^{\mathrm{ej}}$ & $1.38^{\mathrm{dg}}$ & $0.18^{\mathrm{dj}}$ & $1.35^{\mathrm{a}}$ & $0.54^{\mathrm{ac}}$ & $2.59^{\mathrm{bc}}$ & $1.89^{\mathrm{ab}}$ \\
\hline & 100 & $1.37^{\mathrm{eg}}$ & $2.95^{\mathrm{cg}}$ & $4.25^{b c}$ & $3.27^{\mathrm{ad}}$ & $3.09^{\mathrm{ch}}$ & $0.40^{\mathrm{gj}}$ & $2.48^{\mathrm{el}}$ & $1.05^{\mathrm{fg}}$ & $0.09^{\mathrm{ik}}$ & $1.50^{\mathrm{a}}$ & $0.54^{\mathrm{ac}}$ & $2.94^{\mathrm{ac}}$ & $2.04^{\mathrm{ab}}$ \\
\hline & 200 & $2.04^{\mathrm{bf}}$ & $4.03^{\mathrm{a}}$ & $5.35^{\mathrm{a}}$ & $2.67^{\mathrm{bd}}$ & $2.91^{\mathrm{ch}}$ & $0.41^{\mathrm{fj}}$ & $1.34^{\mathrm{jm}}$ & $0.72^{g}$ & $0.08^{\mathrm{jk}}$ & $1.39^{\mathrm{a}}$ & $0.63^{\text {ac }}$ & $2.23^{\mathrm{bc}}$ & $2.02^{a b}$ \\
\hline \multirow[t]{3}{*}{ Bam Wheat cv. } & 50 & $0.10^{\mathrm{h}}$ & $1.72^{\mathrm{hj}}$ & $2.18^{\mathrm{k}}$ & $0.39^{\mathrm{e}}$ & $3.42^{\mathrm{ac}}$ & $1.10^{\mathrm{ab}}$ & $3.87^{\mathrm{ce}}$ & $2.06^{\mathrm{ce}}$ & $0.51^{\mathrm{a}}$ & $1.24^{\mathrm{a}}$ & $0.42^{\mathrm{bc}}$ & $3.00^{\mathrm{ac}}$ & $1.66^{\mathrm{ab}}$ \\
\hline & 100 & $1.24^{\mathrm{eg}}$ & $2.34^{\mathrm{eh}}$ & $2.69^{\mathrm{ek}}$ & $3.60^{\mathrm{ac}}$ & $3.29^{\text {be }}$ & $0.93^{\mathrm{ac}}$ & $3.05^{\mathrm{di}}$ & $1.41^{\mathrm{dg}}$ & $0.35^{b}$ & $1.24^{\mathrm{a}}$ & $0.45^{\mathrm{ac}}$ & $2.74^{\mathrm{ac}}$ & $1.69^{\mathrm{ab}}$ \\
\hline & 200 & $2.69^{b}$ & $3.87^{\mathrm{ab}}$ & $4.90^{\mathrm{ab}}$ & $3.57^{\mathrm{ac}}$ & $2.61^{\mathrm{ci}}$ & $0.75^{\text {bg }}$ & $1.33^{\mathrm{jm}}$ & $0.68^{\mathrm{g}}$ & $0.15^{\mathrm{ek}}$ & $1.28^{\mathrm{a}}$ & $0.56^{\mathrm{ac}}$ & $2.30^{\mathrm{bc}}$ & $1.84^{\mathrm{ab}}$ \\
\hline
\end{tabular}

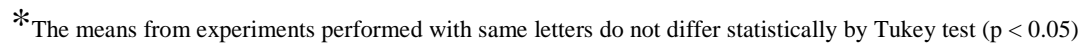

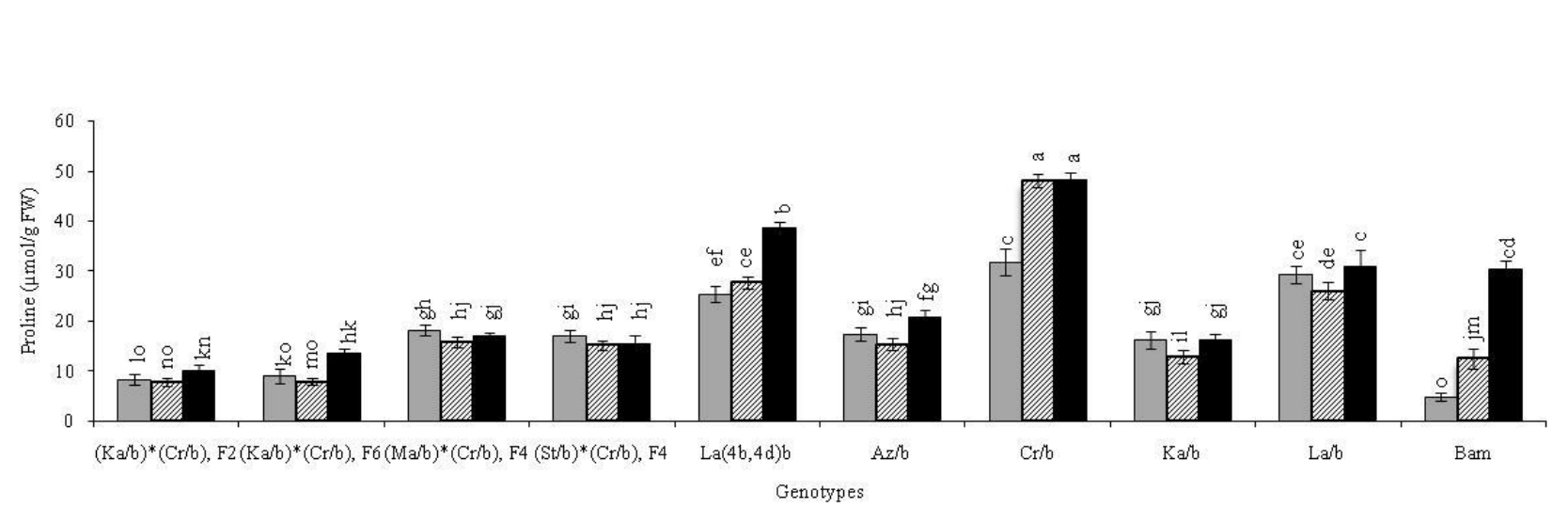

Fig 2. Effect of salinity on flag leaf proline content of primary tritipyrum lines and wheat cultivar Bam. Different letters indicate statistically different means $(\mathrm{p}<0.05)$. 


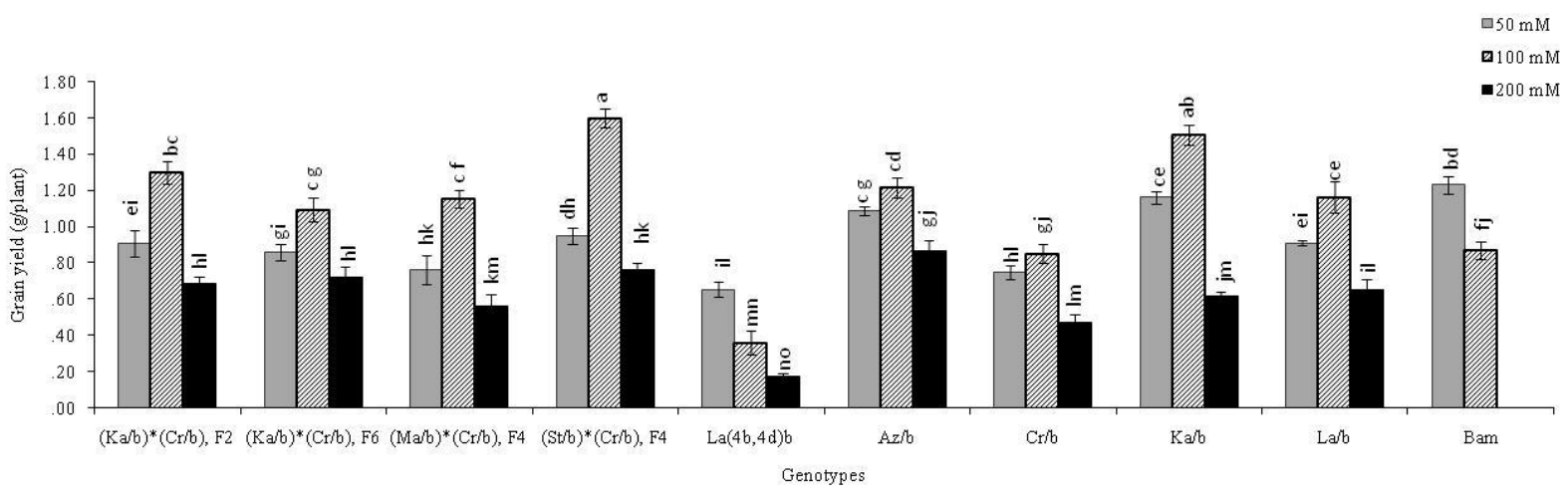

Fig 3. Response of grain yield of primary tritipyrum lines and wheat cultivar bam to salinity treatment. Different letters indicate statistically different means $(\mathrm{p}<0.05)$.

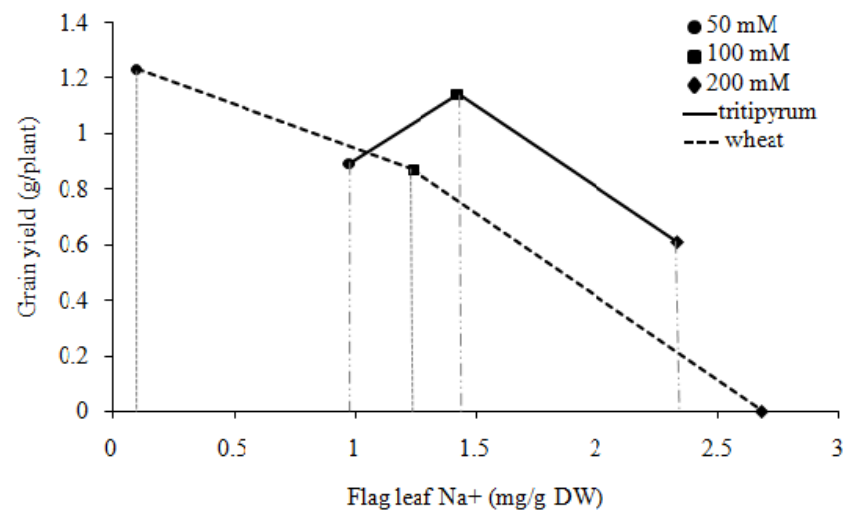

Fig 4. Relationship between flag leaf $\mathrm{Na}^{+}$accumulation on grain yield of tritipyrum lines and wheat cultivar Bam.

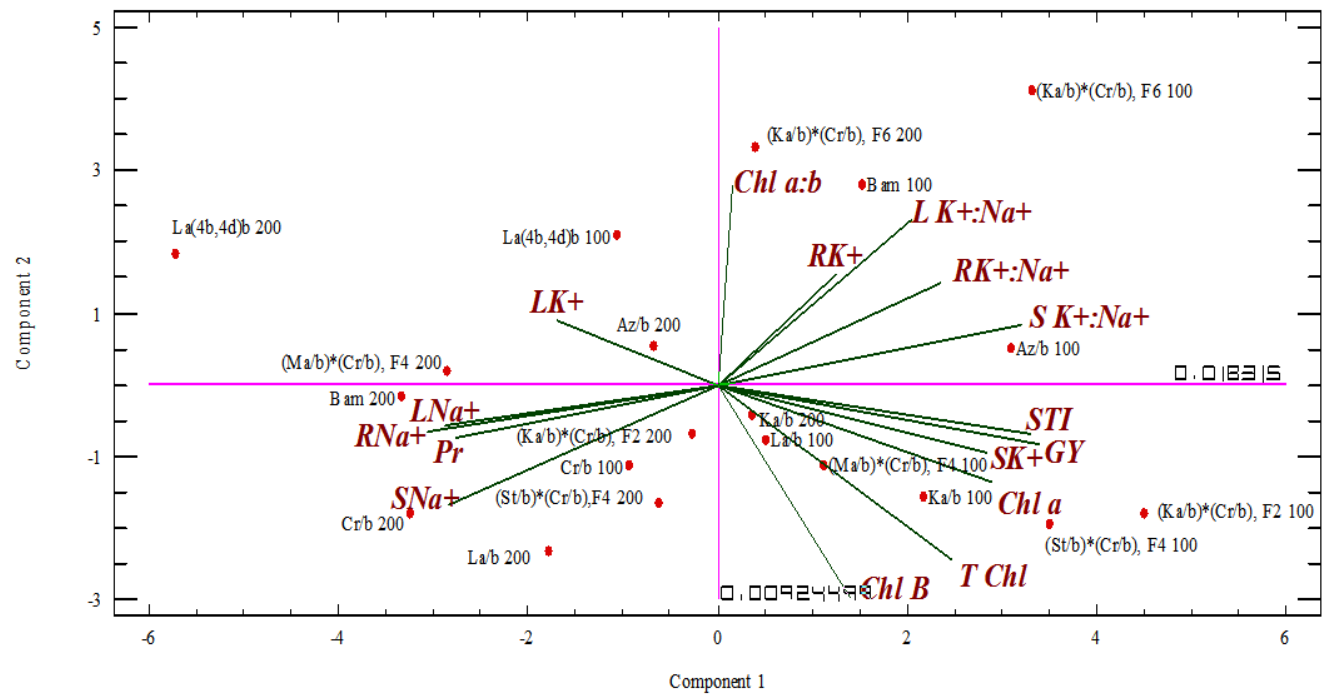

Fig 5. Biplot representation of the results of principal component analysis (PCA) based on measured characteristics of primary tritipyrum lines and wheat cultivar Bam during salinity stress. Genotypes names are beside the red point and the traits are made clear in uppercase letters. LNa: leaf $\mathrm{Na}^{+}$concentration. LK: leaf $\mathrm{K}^{+}$concentration. LK:Na: leaf K/Na ratio. SN: stem $\mathrm{Na}^{+}$concentration. SK: stem $\mathrm{K}^{+}$concentration. SK:Na: stem K/Na ratio. $\mathrm{RN}$ : root $\mathrm{Na}^{+}$concentration. RK: root $\mathrm{K}^{+}$concentration. $\mathrm{RK}: \mathrm{Na}$ : root $\mathrm{K} / \mathrm{Na}$ ratio. Chl a: chlorophyll $a$ concentration. $\mathrm{Chl}$ b: chlorophyll b concentration. Chl $a / b$ : chlorophyll $a$ : chlorophyll $b$ ratio. T Chl: total chlorophyll. GY: grain yield plant ${ }^{-1}$. Pr: proline content. 
and the lowest were related to La (4B, 4D)/b lines (Fig.3). At the highest salt level $(200 \mathrm{mM})$, all primary tritipyrum lines produced grain yield but wheat cultivar Bam did not set seeds. In comparison to grain yield in $50 \mathrm{mM} \mathrm{NaCl}$, primary tritipyrum lines and wheat cultivar Bam presented a $37 \%$ and $100 \%$ reduction in grain yield at $200 \mathrm{mM} \mathrm{NaCl}$, respectively (Fig. 3) and at this salinity level, Az/b produced the highest grain yield, $\mathrm{La}(4 \mathrm{~B}, 4 \mathrm{D}) / \mathrm{b}$ had the lowest and wheat cultivar Bam had no grain yield at all (Fig.3). According to this fact that flag leaf is the major source of assimilates involving in grain filling, the relationship between $\mathrm{Na}^{+}$concentration of flag leaf and grain yield was investigated (Fig. 4). The results showed that increasing salinity caused an increase in grain yield of tritipyrum lines particularly at $100 \mathrm{mM} \mathrm{NaCl}$ while they accumulated higher $\mathrm{Na}^{+}$ions in their flag leaf than wheat cultivar. The stress caused an increase in flag leaf $\mathrm{Na}^{+}$ concentration of wheat and at the same time caused a decline in wheat grain yield and it could not produce any grain at 200 $\mathrm{mM} \mathrm{NaCl}$.

\section{Principal Component Analysis (PCA)}

To analyze the relations among genotypes characteristics, assess the patterns of variation and generally obtain further perception about the physiological mechanisms involved in increasing salt resistance of genotypes, principal component analysis (PCA) was constructed using physiological parameters and grain yield plant $^{-1}$ (Fig. 5). The relationship among measured characteristics during salt treatments and the used genotypes is represented in Fig 5. In this Figure, the correlation coefficient between two traits was estimated by the cosine of the angle between them $\left(\mathrm{r}=\cos 180^{\circ}=-1\right.$, cos $0^{\circ}=1$ and $\cos 90^{\circ}=0$ ).

Five characteristics including $\mathrm{K}^{+}: \mathrm{Na}^{+}$ratio of root, shoot and flag leaf, $\mathrm{K}^{+}$concentration of root and $\mathrm{Chl} \mathrm{a} / \mathrm{b}$ ratio were located in the same quadrant and they were positively correlated to each other. Among these traits, $\mathrm{K}^{+}: \mathrm{Na}^{+}$ratio of stem and root are positively correlated to salinity tolerance index (STI). These traits are related to regulation of ion accumulation and selection of other useful nutrients against $\mathrm{Na}+$ during salinity treatment (Fig. 5). The two dimensional graph of PCA can help improve understanding the relationship among genotypes and measured characteristics. So, this graph can describe the positive role of $\mathrm{K}^{+}: \mathrm{Na}^{+}$ratio of shoot and root on improving salinity tolerance of $\mathrm{Az} / \mathrm{b}$, Bam, $(\mathrm{Ka} / \mathrm{b}) *(\mathrm{Cr} / \mathrm{b}), \mathrm{F}_{6}$ during $100 \mathrm{mM}$ and $(\mathrm{Ka} / \mathrm{b}) *(\mathrm{Cr} / \mathrm{b}), \mathrm{F}_{6}$ in $200 \mathrm{mM} \mathrm{NaCl}$ (Fig. 5). Chlorophyll a, chlorophyll b, total chlorophyll and $\mathrm{K}^{+}$concentration of shoot were located in another quadrant which could suggest the effect of $\mathrm{K}^{+}$ion on photosynthetic apparatus under salinity stress. Their positive relations with salinity tolerance index (STI) showed the positive effect of these traits on performance of genotypes in this quadrant (Fig. 5). $\mathrm{Na}^{+}$content of leaf, shoot and root and proline content were in another quadrant. These traits showed the salinity damages are related to ion toxicity. They were positively correlated with each other but their relations were negative with salinity tolerance index (STI). These relations showed increasing proline and $\mathrm{Na}$ concentration with increasing salinity levels and their negative effect on plant performance of located genotypes in this quadrant (Fig. 5).

\section{Discussion}

Sodium ion is a key ion responsible for the damage caused during salt stress in plants (Munns and Tester, 2008; Tuteja, 2007). Increasing sodium concentration in plant tissues by increasing salinity levels has been observed in different studies (Rajendran et al., 2009; Santa-María and Epstein, 2001). Regarding tritipyrum, the results of our experiments also indicated that salinity increased sodium concentration in various tissues of primary tritipyrum lines and the sodium concentration in different tissues of primary tritipyrum lines was more than that of wheat cultivar Bam (Fig. 1). Despite the higher accumulation of sodium, the yield of primary tritipyrum lines at different levels of salinity was higher than wheat cultivar Bam (Fig. 3). In addition, our results showed that at the second level of applied salinity stress $(100 \mathrm{mM})$, leaf sodium concentration of $(\mathrm{Ma} / \mathrm{b}) *(\mathrm{Cr} / \mathrm{b}), \mathrm{F}_{4}$ and $\mathrm{La} / \mathrm{b}$ lines was approximately equal to leaf sodium concentration of wheat cultivar Bam and leaf sodium concentration of $\mathrm{Ka} / \mathrm{b}$, $(\mathrm{St} / \mathrm{b}) *(\mathrm{Cr} / \mathrm{b}), \mathrm{F}_{4}$ and $(\mathrm{Ka} / \mathrm{b})^{*}(\mathrm{Cr} / \mathrm{b}), \mathrm{F}_{2}$ lines was more than wheat cultivar Bam (Table 2). Nevertheless, the performance of these primary tritipyrum lines was greater than that of wheat cultivar Bam (Fig. 3). It is believed that the increased $\mathrm{Na}^{+}$in plant tissue during salt stress reduces plant performance and there is a negative relationship between wheat leaf sodium concentration and grain yield (Poustini and Siosemardeh, 2004). Therefore sodium exclusion is mentioned as a selection criterion in relation to salt tolerance of cereal crops (Munns et al., 2006). The results of our experiment not only revealed more compatibility and tolerance of primary tritipyrum lines to salinity in comparison with wheat, but also indicated a positive relationship between increasing concentrations of sodium and performance up to $100 \mathrm{mM}$ salinity level. It is consistent with the results of other researchers' experiments who reported the negative effect of $\mathrm{Na}^{+}$on plant performance (Dionisio-Sese and Tobita, 2000; Garthwaute et al., 2005; Munns and James, 2003; Wei et al., 2003). Thus, according to the findings of the present study, salinity tolerance is over shadowed not only by the mechanism of sodium exclusion but also by other involved physiological responses such as "tissue tolerance". In this case the compartmentation of $\mathrm{Na}^{+}$into the vacuoles by a $\mathrm{Na}^{+} / \mathrm{H}^{+}$anti porter may protect the cell from the harmful effect of sodium and the cell uses sodium osmotic adjustment (Blumwald et al., 2000). Primary tritipyrum lines may also have a mechanism of salt tolerance like their ancestor Thinopyrum bessarabicum which uses sodium for osmotic adjustment and thereby increases its tolerance to salinity (Colmer et al., 2006b).

Despite higher sodium concentration of $\mathrm{La}(4 \mathrm{~B}, 4 \mathrm{D}) / \mathrm{b}$ primary tritipyrumin than wheat cultivar Bam at $200 \mathrm{mM}$ salinity level, this line had the lowest performance while wheat cultivar Bam had no grain yield (Figs. 3). These observations may imply a strong system of tissue tolerance in $\mathrm{La}(4 \mathrm{~B} / 4 \mathrm{D}) / \mathrm{b}$. It is worth mentioning that $\mathrm{D}$ genome includes Kna1 locus on the long arm of chromosome $4 \mathrm{~d}$ that leads to lower sodium concentration (Dubcovsky et al., 1996; Gorham et al., 1987). Nevertheless, higher concentration of sodium in $\mathrm{La}(4 \mathrm{~B} / 4 \mathrm{D}) / \mathrm{b}$ line's leaf tissue and its lower performance than that of other primary tritipyrum lines may be the result of either negative interaction between $\mathrm{D}$ genome with $\mathrm{A}$ genome of durum wheat and $\mathrm{E}_{\mathrm{b}}$ genome of Th. bessarabicum that leads to decreased expression of this trait on $\mathrm{D}$ genome or the other genes brought about by $\mathrm{D}$ genome that increase sodium when combined with the other genes of this line. Overall, the results regarding the concentration of sodium ions in primary tritipyrum lines showed a correlation between tissue tolerance and sodium exclusion mechanisms under salinity stress which might play a key role in salinity tolerance of primary tritipyrum lines.

Potassium is mentioned as an essential monovalent cation that has a positive effect on improving salt tolerance in plants (Cuin et al., 2008; Munns et al., 2006; Rascio et al., 2001). 
Salt tolerance is associated with transport of potassium from root to shoot (Chen et al., 2005; Chen et al., 2007; Cuin et al., 2008).

In the present study, potassium content of different tissues of primary tritipyrum lines at different salinity levels did not follow a specific pattern, but in wheat cultivar $\mathrm{Bam} \mathrm{K}^{+}$ concentration decreased with increasing salinity levels (Fig. 1). In addition, fluctuations in potassium concentration at various salinity levels were not as high as that of sodium (Fig. 1). Although the amount of potassium in different tissues and at different salinity levels of wheat cultivar Bam was higher than that of primary tritipyrum lines, its performance was more affected by salinity (Fig. 1). Unlike $\mathrm{Na}^{+}$concentration, $\mathrm{K}^{+}$concentration increased from root to shoot and leaves in studied genotypes. The concentration of potassium in different tissues can be a sign of other involved physiological mechanisms that, despite the accumulation of high levels of sodium and very little changes in potassium, maintains ionic balance and therefore increases salinity tolerance. It can be noted that maintaining the potassium levels while salinity is increasing may be hereditary as primary tritipyrum ancestor, Th. bessarabicum, that preserves $\mathrm{K}^{+}$concentration in salinity conditions as another strategy to increase tolerance to salinity stress (Colmer et al., 2006b).

Several studies suggest a positive relationship between the $\mathrm{K}^{+} / \mathrm{Na}^{+}$ratio and high salt tolerance. Thus, this ratio is used as another selection criterion for the selection of resistant varieties (Chen et al., 2007). According to the results in this experiment, there is a positive relationship between this ratio and stress tolerance (Fig. 5) and it seems that this ratio can be assumed as a suitable criterion for determining resistance to salt stress in this plant.

At all salinity levels, the amount of chlorophyll $a$ and chlorophyll $b$ of primary tritipyrum lines were higher than that of wheat cultivar Bam. However, an increase in the salinity level reduced chlorophyll $a$ and $b$ in both primary tritipyrum lines and wheat cultivar Bam. But this reduction was higher in wheat than primary tritipyrum lines (Table 2). In general, chlorophyll content had a positive effect on grain yield plant ${ }^{-1}$ under salinity treatment (Fig. 5). Experiments reported greater drop of chlorophyll $a$ and $b$ in a salt sensitive wheat variety than salt tolerant variety and suggested that the increase in Chl $a / b$ implied that photosystem II was affected more by salinity (El-Shintinawy, 2001; Zheng et al., 2008). Thus, it seems that the prevention of the chlorophyll biosynthesis and the degradation of chlorophyll are the main factors for this result (Husain et al., 2003; Khan, 2003; Khatkar and Kuhad, 2000).

In our experiment, there was a positive relation between proline and $\mathrm{Na}^{+}$concentration in leaf, stem and root (Fig. 5). In other words, increasing $\mathrm{Na}^{+}$caused increase in proline accumulation (Fig. 5). Furthermore, proline showed negative relationship with salinity tolerance index (STI) and grain yield plant ${ }^{-1}$ (Fig. 5). These observations can be related to the fact that, proline accumulation can be a sign of stress injury and it cannot be a protective compound against injurious effects of high concentration of ions during salinity stress and its role in osmotic adjustment either is lower than other ions or organic solutes and maybe it does not play any role in osmotic adjustment. The reports about the role of proline on salinity tolerance are different. Some researchers indicated that there are no relations between proline content and salinity tolerance (Lutts et al. 1996; Poustini et al., 2007). Lutts et al.(1996) found no relationship between proline content and salt tolerance in rice cultivars. It was concluded that proline may not be involved in salt tolerance in wheat cultivar (Poustini et al., 2007). However, other reports indicated that proline acts as osmoprotectant and causes salt tolerance (Ashraf and Foolad, 2007; Wagdy et al., 2002; Goudarzi and Pakniyat, 2009). In some cases, proline was introduced as a biochemical marker for increasing salt tolerance (Ashraf and Harris, 2004; Martinez et al., 1996). Tritipyrum lines may use $\mathrm{Na}^{+}$ions as an osmotic adjustment agent similar to its ancestor Thinopyrum bessarabicum (Colmer et al., 2006b). The negative relation between proline content and grain yield plant ${ }^{-1}$ signified that proline has no direct role in the salt tolerance of the used genotypes.

\section{Materials and methods}

\section{Genetic materials}

Seeds were kindly provided by Dr. Shahsavand (Shiraz University, Iran). Tritipyrum lines included $(\mathrm{Ka} / \mathrm{b}) *(\mathrm{Cr} / \mathrm{b})$, $\mathrm{F}_{6},(\mathrm{Ka} / \mathrm{b}) *(\mathrm{Cr} / \mathrm{b}), \mathrm{F}_{2},(\mathrm{Ma} / \mathrm{b})^{*}(\mathrm{Cr} / \mathrm{b}), \mathrm{F}_{4},(\mathrm{St} / \mathrm{b}) *(\mathrm{Cr} / \mathrm{b}), \mathrm{F}_{4} \mathrm{La}$ (4B, 4D)/b, St/b, Cr/b, Az/b, La/b, Ka/b (Table 1). T.aestivum cv. Bam (salt tolerant) and T. aestivum cv. Gascoigne (salt sensitive) were used as standard based on findings of other studies carried out on their salt tolerance (Vahabzadeh et al., 2009).

\section{Verbalization method}

Seeds that were uniform in shape and weight were selected. The selected seeds were sown in seedling growing trays (with $2 \mathrm{~cm}$ in diameter and $7 \mathrm{~cm}$ deep cells) which were filled with sand, soil and manure (2:1:1). Then they were vernalized for 6 weeks outside of the green house. At the end of the sixth weeks, the seedlings were at $3^{\text {rd }}$ leaf stage and ready for being transplanted in planting containers $(43 \times 34 \times 25 \mathrm{~cm})$.

\section{Plants growth condition}

The experiment was conducted in a glasshouse in the Faculty of Agriculture, Ferdowsi University of Mashhad, Iran, with daily temperature of $25^{\circ} \mathrm{C} / 20^{\circ} \mathrm{C}$ day/night and natural light without relative humidity control in a hydroponic culture system. A drain was placed in the bottom of planting containers, and then they were filled to a depth of $5 \mathrm{~cm}$ with gravels that had a diameter of one centimeter. Then, rest of the space in the containers was filled with washed sand to a depth of $23 \mathrm{~cm}$. The containers were kept on iron benches with a height of $80 \mathrm{~cm}$ above ground level. Three irrigation pipes with a length of $40 \mathrm{~cm}$ and a diameter of $5 \mathrm{~mm}$ were placed in $10 \mathrm{~cm}$ spacing between them in each container. Holes for water withdrawal were inserted on any irrigation pipe that was buried at the depth of $2 \mathrm{~cm}$ below the sands in the container. The pipes in each container were connected to a main water pipe and irrigation was done through a pump attached to it. Pumps were inserted in 100 liter plastic barrels which were located on the floor near the iron bench. Healthy and similar seedlings in shape and size were selected and transferred to the containers. In each container, 4 rows of seedlings were planted according to a planting pattern with 8 $\mathrm{cm}$ spacing between rows and $2 \mathrm{~cm}$ spacing within rows. The seedlings were irrigated with half strength Hoagland solution (Hoagland 1950). After three days, the seedlings received full strength Hoagland solution and $\mathrm{pH}$ of the solution was maintained at 6.0 with $5 \mathrm{~N} \mathrm{HNO}$. The electrical conductivity of the solution in each barrel was controlled twice a week. Since evaporation caused a loss in the volume of water in the barrel, the amount of water lost from the barrel was replaced by holding its volume up to 100 liters. Salt treatments were applied incrementally at the $4^{\text {th }}$ leaf stage. To perform salt 
treatments, $\mathrm{NaCl}$ was added in 3 steps by $50 \mathrm{mM} /$ day to reach the intended salinity levels, i.e. 50, 100 and 200 $\mathrm{mMNaCl}$. Control plants received $50 \mathrm{mM} \mathrm{NaCl}$. All plants were watered daily by pumping Hoagland's solution containing 50,100 and $200 \mathrm{mM} \mathrm{NaCl}$ and allowed it to be drained into the holding barrels. The solution was renewed weekly.

\section{Tissues $\mathrm{Na}^{+}$and $\mathrm{K}^{+}$measurement}

Seven plants of each treatment were harvested at $50 \%$ ear emergence stage of each genotype. Then the root, stem and flag leaf blade of each genotype were separated and used to measure the concentration of sodium and potassium ions (Isaac and Kerber, 1971). Flag leaf blades were washed by distilled water because it was likely that the surfaces of leaves were coated with salt inadvertently. The flag leaf blades, as well as sheets were placed in small paper envelopes and dried in an oven at $60^{\circ} \mathrm{C}-70^{\circ} \mathrm{C}$ for 2 days.

Roots were rinsed rapidly to prevent efflux of $\mathrm{Na}^{+}$from root cells. Washed roots were put into small paper envelops and dried in an oven at $60^{\circ} \mathrm{C}-70^{\circ} \mathrm{C}$ for two days. Dried roots, shoots and flag leaf blade were ground by an electric mill. One gram of the milled tissue was heated in an electric furnace at a temperature of $580^{\circ} \mathrm{C}$ for two hours. To release cations, the produced ash was washed by $2 \mathrm{M}$ hydrochloric acid. The extract was diluted with distilled water and filtered with a filter paper and sodium and potassium concentrations were measured with a flame photometer.

\section{Chlorophyll measurement}

To measure the amount of chlorophyll, Lichtentaler method (1987) was applied. 0.1 gram of leaf tissue was ground in a mortar and mixed with $4 \mathrm{ml}$ of $80 \%$ acetone. The resulting solution was centrifuged for 5 minutes at $3000 \mathrm{rpm}$. To determine the amount of chlorophyll, the absorption of the supernatant was read with spectrophotometer at a wavelength of $647,664 \mathrm{~nm}$. The total chlorophyll, and chlorophyll $a$ and $b$ were calculated by the following equations:

$$
\begin{aligned}
& C h l_{b}=21.21 A_{647}-5.1 A_{664} \\
& C h l_{a}=12.25 A_{664}-2.79 A_{647} \\
& C h l_{T}=C h l_{a}+C h l_{b}
\end{aligned}
$$

Where, $\mathrm{A}_{647}$ and $\mathrm{A}_{664}$ are absorbance at 647 and $664 \mathrm{~nm}$ wave lengths, respectively. $\mathrm{Chl}_{\mathrm{a}}$ is chlorophyll $a, \mathrm{chl}_{\mathrm{b}}$ is chlorophyll $b$ and $\operatorname{chl}_{\mathrm{T}}$ is the total chlorophyll.

\section{Proline content of flag leaf tissue}

The flag leaf of each genotype grown under salt stress conditions were sampled at $50 \%$ ear emergence stage and kept at $-80^{\circ} \mathrm{C}$ until use. 0.5 gram of frozen leaf tissue was ground in liquid nitrogen. Proline was extracted with $10 \mathrm{ml}$ $3 \%(w / v)$ sulfosalicylic acid. After centrifugation, the supernatant was used for determination of proline content as described by Bates et al (1973). Two milliliter of the extract was reacted with $2 \mathrm{ml}$ glacial acetic acid and 2 milliliter ninhydrin reagent in a test tube and was incubated at $100^{\circ} \mathrm{C}$ for one hour. The mixture was cooled in an ice bath to terminate the reaction and then it was translocated to room temperature. $2 \mathrm{ml}$ toluene was added to the reaction mixture and was mixed vigorously for 15-20 seconds. The chromophore phase containing toluene was collected and its absorbance was recorded at $520 \mathrm{~nm}$ using toluene as blank.
Proline concentration was determined using standard curve as $\mu \mathrm{g} / \mathrm{ml}$ and calculated on fresh weight basis as follows: $[((\mu \mathrm{g}$ proline $) / \mathrm{ml} \times \mathrm{ml}$ toluene $) /(115.5 \mu \mathrm{g}) /$ $(\mu$ mole $)] /[(($ g sample $)) / 5]=(\mu$ moles proline $) /$ ( $\mathrm{g}$ fresh weight)

\section{Grain yield Measurement}

Grain yield per plant was measured by weighing grains of all plant tillers at the maturity stage.

Salt tolerant index (STI) was calculated according to Fernandez (1992),

$\mathrm{STI}=((\mathrm{Yp} \cdot \mathrm{Ys})) /(\mathrm{Yp})^{\wedge} 2$

Where Ys is the yield of genotype in stress condition and Yp is the yield of genotypes under control condition.

\section{Experimental design and statistical analysis}

Experimental design was split plot in completely randomized design with three replications. Simple statistical analysis including mean comparison and standard error were conducted by SPSS (Ver. 16.0, SPSS, Chicago, IL, USA). Mean comparisons were calculated using Tukey's test at 5\% probability level. Principal component analysis (PCA) was used to obtain a multivariate view of collected variables and data. Principal component analysis (PCA) and cluster analysis were carried out using Statgraphics Centurion version 16 software. Charts were drawn using Excle and Sigmaplot 10 software.

\section{Conclusion}

The results of this experiment indicated that primary tritipyrum lines showed more salt tolerance than wheat salt tolerant cultivar Bam. It seems that after solving some agronomical problems related to this plant such as fragile rachis and improving its quantitative and qualitative traits, the plant will have the capability of being used in saline lands to improve this lands productivity. Moreover, by understanding the physiological mechanisms of salt stress tolerance in tritipyrum lines and identifying genes involved, due to the proximity of this plant to wheat, primary tritipyrum lines can be used to increase the salt tolerance of wheat varieties and enhance its performance under salt stress. Unlike wheat, primary tritipyrum lines are young and new cereal crop; therefore this preliminary study could be a prelude to a longterm project to increase salt tolerance in wheat.

\section{Acknowledgements}

The authors would like to express their appreciation to Ferdowsi University of Mashhad, Iran, for providing funds of this research.

\section{References}

Apse MP, Blumwald E (2007) $\mathrm{Na}^{+}$transport in plants. Febs Lett. 581: 2247-2254.

Ashraf M, Foolad M (2007) Roles of glycine betaine and proline in improving plant abiotic stress resistance. Environ Exp Bot. 59(2): 206-216.

Ashraf M, Harris P (2004) Potential biochemical indicators of salinity tolerance in plants. Plant Sci. 166(1): 3-16.

Bates L, Waldren R, Teare I (1973) Rapid determination of free proline for water-stress studies. Plant soil. 39: 205-207.

Blumwald E, Aharon GS, Apse MP (2000) Sodium transport in plant cells. BBA- Biomembranes. 1465: 140-151. 
Chen Z, Newman I, Zhou M, Mendham N, Zhang G, Shabala S (2005) Screening plants for salt tolerance by measuring $\mathrm{K}+$ flux: a case study for barley. Plant Cell Environ. 28: 12301246.

Chen Z, Zhou M, Newman IA, Mendham NJ, Zhang G, Shabala $S$ (2007) Potassium and sodium relations in salinised barley tissues as a basis of differential salt tolerance. Funct Plant Biol. 34: $150-162$

Colmer T, Munns R, Flowers T (2006a) Improving salt tolerance of wheat and barley: future prospects. Anim Prod Sci. 45: 1425-1443.

Colmer TD, Flowers TJ, Munns R (2006b) Use of wild relatives to improve salt tolerance in wheat. J Exp Bot. 57: 1059-1078.

Cuin TA, Betts SA, Chalmandrier R, Shabala S (2008) A root's ability to retain $\mathrm{K}+$ correlates with salt tolerance in wheat. $\mathrm{J}$ Exp Bot. 59: 2697-2706.

Dionisio-Sese ML, Tobita S (2000) Effects of salinity on sodium content and photosynthetic responses of rice seedlings differing in salt tolerance. J Plant Physiol. 157: 54-58.

Dubcovsky J, Santa Maria G, Epstein E, Luo MC, Dvořák J (1996) Mapping of the $\mathrm{K}+/ \mathrm{Na}+$ discrimination locus Kna1 in wheat. Theor Appl Genet. 92: 448-454.

El-Shintinawy F (2001) Photosynthesis in two wheat cultivars differing in salt susceptibility. Photosynthetica. 38: 615-620.

Faris JD (2014) Wheat domestication: Key to agricultural revolutions past and future. Tuberosa R, Graner A, Frison E (eds) Genomics of Plant Genetic Resources. Springer, Houten Netherlands, p 439.

Flowers TJ, Colmer TD (2008) Salinity tolerance in halophytes. New Phytol. 179: 945-963.

Garthwaite AJ, von Bothmer R, Colmer TD (2005) Salt tolerance in wild Hordeum species is associated with restricted entry of $\mathrm{Na}+$ and $\mathrm{Cl}-$ into the shoots. J Exp Bot. 56: 2365-2378.

Genc Y, Mcdonald GK, Tester M (2007) Reassessment of tissue $\mathrm{Na}+$ concentration as a criterion for salinity tolerance in bread wheat. Plant Cell Environ. 30: 1486-1498.

Gorham J, Hardy C, Jones RW, Joppa L, Law C (1987) Chromosomal location of a $\mathrm{K} / \mathrm{Na}$ discrimination character in the D genome of wheat. Theor Appl Genet. 74: 584-588.

Gorham J, McDonnell E, Budrewicz E, Jones RW (1985) Salt tolerance in the Triticeae: growth and solute accumulation in leaves of Thinopyrum bessarabicum. J Exp Bot. 36: 10211031.

Goudarzi M, Pakniyat H (2009).Salinity causes increase in proline and protein contents and peroxidase activity in wheat cultivars. J Appl Sci. 9(2): 348-353.

Hoagland DR, Arnon DI (1950) The water-culture method for growing plants without soil. Circular. Calif AES Bull. p 347.

Husain S, Munns R, Condon AT (2003) Effect of sodium exclusion trait on chlorophyll retention and growth of durum wheat in saline soil. Crop Pasture Sci. 54: 589-597.

Isaac R.A., Kerber J.D. (1971): Atomic absorption and flame photometry: Techniques and uses in soil, plant and water analysis. In: Instrumental Methods for Analysis of Soil and Plant Tissue. Walsh, L. M. (Ed.). Soil Science of America, Madison, Wisconsin, pp. 17-37.

Khan N (2003) NaCl-inhibited chlorophyll synthesis and associated changes in ethylene evolution and antioxidative enzyme activities in wheat. Biol Plantarum. 47: 437-440.

Khatkar D, and Kuhad M (2000) Short-term salinity induced changes in two wheat cultivars at different growth stages. Biol Plantarum. 43: 629-632.
King I, Law C, Cant K, Orford S, Reader S, Miller T (1997) Tritipyrum, a potential new salt-tolerant cereal. Plant Breeding. 116: $127-132$.

Lichtenthaler HK (1987) Chlorophylls and carotenoids: pigments of photosynthetic biomembranes. Method Enzymol. 148: 350382.

Lutts S, Kinet J, Bouharmont J (1996). Effects of salt stress on growth, mineral nutrition and proline accumulation in relation to osmotic adjustment in rice (Oryza sativa L.) cultivars differing in salinity resistance. Plant Growth Regul. 19(3): 207-218.

Martinez C A, Maestri M, Lani EG (1996) In vitro salt tolerance and proline accumulation in Andean potato (Solanum spp.) differing in frost resistance. Plant Sci. 116(2): 177-184.

Mudgal V, Madaan N, Mudgal A (2010) Biochemical mechanisms of salt tolerance in plants: a review. Int $\mathrm{J}$ Bot. 6: 136-143.

Munns R, and James RA (2003) Screening methods for salinity tolerance: a case study with tetraploid wheat. Plant Soil. 253: 201-218.

Munns R, James RA, Läuchli, A (2006) Approaches to increasing the salt tolerance of wheat and other cereals. J Exp Bot. 57: 1025-1043.

Munns R, Tester M (2008) Mechanisms of salinity tolerance. Annu Rev Plant Biol. 59: 651-681.

Parida AK, Das AB (2005) Salt tolerance and salinity effects on plants: a review. Ecotox Environ Safe. 60: 324-349.

Peng JH, Sun D, Nevo E (2011) Domestication evolution, genetics and genomics in wheat. Mol Breeding. 28: 281-301.

Poustini K, Siosemardeh A (2004) Ion distribution in wheat cultivars in response to salinity stress. Field Crops Res. 85: 125-133.

Poustini K, Siosemardeh A, Ranjbar M (2007) Proline accumulation as a response to salt stress in 30 wheat (Triticum aestivum L.) cultivars differing in salt tolerance. Genet Resour Crop Ev. 54(5): 925-934.

Rajendran K, Tester M, Roy, SJ (2009) Quantifying the three main components of salinity tolerance in cereals. Plant Cell Environ. 32: 237-249.

Rascio A, Russo M, Mazzucco L, Platani C, Nicastro G, Di Fonzo N (2001) Enhanced osmotolerance of a wheat mutant selected for potassium accumulation. Plant Sci. 160: 441-448.

Santa-María GE, Epstein E (2001) Potassium/sodium selectivity in wheat and the amphiploid cross wheat X Lophopyrum elongatum. Plant Sci. 160: 523-534.

Sawahel WA, Hassan AH (2002) Generation of transgenic whea plants producing high levels of the osmoprotectant proline. Biotechnol Lett. 24(9): 721-725.

Tuteja N (2007) Mechanisms of high salinity tolerance in plants. Method enzymol. 428: 419-438.

Vahabzadeh M, Heravan EM, Meybodi HH, Tabatabaie M, Bozorgipour R, Bakhtiar F, Akbari A, Pakdel A, Sharifalhosseini M, Afyoni D (2009) Bam, A New Bread Wheat Cultivar for Moderate Climate Zones with Salinity of Soil and Water. Seed and Plant Improvment Journal. 25: 223 226.

Wei W, Bilsborrow PE, Hooley P, Fincham DA, Lombi E, Forster BP (2003) Salinity induced differences in growth, ion distribution and partitioning in barley between the cultivar Maythorpe and its derived mutant Golden Promise. Plant Soil. 250: 183-191.

Zheng Y, Wang Z, Sun X, Jia A, Jiang G, Li Z (2008) Higher salinity tolerance cultivars of winter wheat relieved senescence at reproductive stage. Environ Exp Bot. 62: 129-138. 